\title{
JARDINS ET PALAIS DANS LE CORAN ET LES MILLE ET UNE NUITS.
}

\author{
Edgard WEBER \\ Université Toulouse-Le Mirail
}

Je voudrais exprimer, à travers ces quelques pages, toute mon amitié à María Jesús Rubiera Mata, qui, entre ses premiers écrits de 1966 aux plus récents de 1993, a publié pas moins de cent douze articles et plusieurs livres d'une très haute qualité scientifique. De la littérature arabe en général, en passant par l'histoire de Grenade et d'Al-Andalus, l'étude des toponymes hispano-arabes, l'architecture arabe..., María Jesús nous conduit jusqu'aux Mille et Une Nuits dont elle a si intelligemment éclairé maints aspects. Moi-même amateur des contes arabes, $j$ 'ai suivi avec un plaisir immense cette approche savante et fine de certaines Nuits. Grande connaisseuse des Mille et Une Nuits ${ }^{1}$, elle l'est aussi, entre autres, de l'architecture arabo-musulmane ${ }^{2}$. C'est

${ }^{1}$ Les travaux se rapportant à la littératurc et aux contes: "La mesa de Salomón", in Awraq, 3 (1980), pp. 55-64, et "Le module de la Cité Pétrifiée et sa présence dans la littérature hispanique", Les Mille et Une Nuits. Contes sans frontière, Toulouse, AMAM, 1994, pp. 221-227.

"Antigua literatura árabe", in Historia Universal de la literatura, Orbis, 48, Barcelona, 1983, 17 p.

${ }^{2}$ Les études propres à l'architecture arabo-musulmane: La arquitectura en la literatura árabe. Datos para una estética del placer, Editora Nacional, Madrid, 1981, 181 p. Segunda edición aumentada, Madrid, ed. Hiperión, 1988, 196 p.

"La descripción poética de los palacios árabes: datos para la definición del género "qusūriyyät»", in Actas del IV Coloquio Hispano-Tunecino, Palma de Mallorca, 1979, Instituto Hispano-Árabe de Cultura, Madrid, 1983, pp. 213-215.

"La función estética del agua en la civilización arabigoislámica". Agua y poblamiento musulmán/Aigua i poblament musulmà, Ajuntament de Benissa, 
donc vers ces deux spécialités que ces quelques pages seront adressées. En tous cas, je lui dois d'avoir découvert le rapport entre les Nuits et l'architecture arabo-musulmane.

En effet, dans un grand nombre de contes, il est question de jardins dans lesquels se dresse généralement un merveilleux palais; il y est aussi question de villes dont le conteur décrit avec finesse l'architecture. Je croyais d'abord que ces descriptions des jardins et des palais, voire des personnages qui y habitaient, n'étaient qu'une pure convention qui n'ajoutait rien d'important à l'histoire qui se déroulait sur les lèvres du conteur et dont les auditeurs se délectaient avec joie. Mais une lecture plus attentive, plus minutieuse, telle que María Jesús nous en donne toujours un éloquent exemple, m'a permis de replacer ces descriptions dans une stratégie "contique" beaucoup plus importante, une stratégie qui consiste à mobiliser l'attention de l'auditoire, en évoquant cela même qui constitue la fine fleur de l'imaginaire arabo-musulman: le paradis céleste. Quel rôle joue dès lors la description des jardins et des palais dans Les Mille et Une Nuits, sinon celui de porter l'auditeur, à travers cette description même, jusqu'au seuil de ce qui fera plus tard son bonheur absolu et dont il entrevoie, hic et nunc, les premiers frémissements, à travers une histoire qui parle plus profondément à son inconscient qu'à la raison?.

Le theme du jardin, dans Les Mille et Une Nuits prend donc une dimension et une importance toute particulière si l'on songe que dans l'imaginaire arabo-musulman, sa mention, a plus forte raison sa description luxurieuse et luxuriante, ne peut que renvoyer l'auditeur a la mention du jardin céleste si abondamment rappelé dans le Coran comme récompense de ceux qui font le bien sur terre. Tout se passe comme si, à travers une histoire plaisante, mise fictivement dans la bouche d'une femme, le conteur brodait sur un theme bien connu. Quelques mots, quelques expressions, quelques allusions suffisent pour passer des plaisirs terrestres des Mille et Une Nuits aux plaisirs célestes évoqués par le Coran. Faut-il aller jusqu'à dire que certains passages des Mille et Une Nuits sont en quelque sorte un commentaire libre de

Benissa, 1988, pp. 11-12. L'immaginario el'archittetura nella letteratura araba medievale, traduction de La arquitectura en la literatura árabe, edition y prologue de Ennio Concina, Génova, ed. Marietti, 1990, 163 pp. 
certains versets du Coran, à la manière d'un lointain ${ }^{3}$ midras agada ${ }^{4}$ que les rabbins manipulaient avec art pour expliquer certains livres du Pentateuque? Cette manière d'actualiser la Bible, dans la tradition juive, pouvait donner lieu à des commentaires édifiants mais aussi, et bien souvent, amusants. Le but pédagogique de la agada est en effet de divertir tout en instruisant le fidele. Parfois considéré comme pure "légende", en raison de la liberté qu'il prend par rapport au texte qu'il est sensé expliquer, le midraš reste néanmoins "legenda" au sens latin, à savoir une lecture et une leçon. Certes, il ne faudrait pas prendre les Nuits comme un midraš agada du Coran. Mais voyons seulement que lorsqu'il s'agit de bonheur absolu, les Nuits rejoignent curieusement les promesses du Coran.

Dans le monde arabo-musulman, comme dans certaines civilisations antérieures ${ }^{5}$, le jardin est un espace mythique et réel à la fois où

${ }^{3}$ En effet, le conteur des Mille el Une Nuits ignore sans doute la véritable pratique midrashique. Néanmoins, utilise-t-il des traditions qui remontent à l'élaboration du hadịt et de la sira, aux influences des isrä iliyyät, enfin aux commentaires coraniques marqués par la littérature rabbinique comme dans la géniale et immense compilation du tafsir de Tabarī $(\mathrm{m} .923)$ dont s'inspireront tant d'auteurs postérieurs. Dans tous ces apports, le souvenir du midrăš ne doit pas être écarté trop rapidement. Cf. par exemple les traditions auxquelles se rapporte al-Muhāsibī (m. 857) pour décrire le bonheur des bienheureux au Paradis, dans son Kităb al-tawahhum, éd. Arberry, Le Caire, 1937, traduction par A. Roman, Klincksieck, 1978.

${ }^{4}$ On peut considérer la agada comme un enjolivement du Texte sacré. Le midraš est un procédé exégétique pratiqué par les rabbins sur la Thora qui consiste plus en une paraphrase libre qu'une étude "scientifique" au sens moderne. L'importance de la littérature midrašique à l'époque tanaïtique (Ier et IIème siècle ap. J.C.) est très grande. Les textes chrétiens primitifs en sont indubitablement marqués. N'oublions jamais que les premiers "chrétiens" ne sont que des Juifs "dissidents" par rapport au judaïsme officiel. La littérature midrašique continue les siècles suivants jusqu'à l'apparition de l'islam (VIIème siècle ap. J.C), voire même au-delà. Or parmi les premiers musulmans, il y aura encore des Juifs (marqués par cette lecture possible des Textes sacrés) et des chrétiens... Pour avoir une idée de cette littérature: Taradach, Madeleine, Le Midrash, introduction à la littérature midrashique, Labor et Fides, 1991 (avec une abondante bibliographie).

${ }^{5}$ Déjà dans l'épopée de Gilgamesh (2700 av. J.C.) à Sumer, il est question d'un arbre au tronc en or, décoré de pierres précieuses: émeraudes et joyaux 
l'homme vient goûter le bonheur. C'est pour cette raison qu'il a toujours eu une importance capitale autant dans la littérature édifiante que dans ses nombreuses variations architecturales, que ce soient les premiers vestiges à Bagdad et Sāmarrā ou les magnifiques bagh (jardins-palais) de Samarkand ${ }^{6}$, en passant par les agdal des villes impériales ${ }^{7}$ du Maroc, l'Alhambra de Grenade, les jardins safavides ${ }^{8}$ de l'lran ou même plus loin encore les jardins moghols ${ }^{9}$ de l'Inde et du Cachemir. Sans doute, dès la plus haute antiquité, en a-t-il été ainsi avec les jardins suspendus de Babylone, avec ceux que la reine Hatshepsout (1520-1484 av. J.C.) ou Ramsès III (1198-1166 av. J.C.) dédiaient à Amon et ceux que le jeune Cyrus (424-401 av. J.C.), après Darius le Grand, élevait au rang d'un véritable art aux dires de Xénophon (430-355 av. J.C). Mais il faudra sans doute attendre les Sassanides ${ }^{10}$, grands amateurs de jardin, pour que l'image de celui-ci

rares. Derrière cet arbre se trouve la source sacrée... Pr ' 'mière attestation du mythe du Paradis qui connaîtra un si grand développement dans la tradition monothéiste, dans le judaïsme el l'islam surtout. Le plus surprenant est que cette représentation de l'arbre magique se retrouve dans une miniature du Mi'rāğ Nāmeh de 1436 ap. J.C.!

${ }^{6}$ Tamerlan (m. 1405) entoura Samarkand d'une véritable ceinture de jardins dont le fameux Shahar bagh (Quatre jardins). Après lui, les souverains timourides entretiennent cette tradition. Ainsi, le moghol Bābur (XVème. s.) en orne-t-il Kabul avant de s'installer à Agra. Le Bābur-nāme réunit de superbes miniatures du fameux Bagh vafa (Jardin de fidélité) près de Jalalabad. Son successeur Humayūn en édifia un à Delhi où il est enterré.

${ }^{7}$ Surtout ceux de Meknès et de Marrakech. Cf. G. Marçais, "Les Jardins de l'Islam", in Mélanges d'histoire et d'archéologie de l'Occident Musulman, I, Paris, 1957.

${ }^{8}$ Les Safavides et notamment Shah Abbās le Grand embellirent leur capitale Isphahan où le palais des réceptions Ali Qapu, le palais aux Quarante colonnes, le palais des Huit paradis subsistent encore d'un complexe qui réunissait jardins, palais, bassins et fontaines.

${ }^{9}$ Notamment ceux de l'empereur Akbar (m. 1605) et de ses descendants Humayūn, Ğihānğir et Shāh Ğihān dont le plus grand est Shalamar. C'est Bābur qui introduisit en Inde les bagh d'origine iranienne.

${ }^{10}$ Dynastie perse qui règne du IIIème au VIIème s. à laquelle la conquête arabe a mis fin, non sans en recueillir des influences de tous ordres. Le grand poète persan Ferdowsi (939-1020) auteur du Š̂h-nâme (Le Livre des Rois) et le 
évoque le plus directement le paradis de la révélation muhammadienne. Notons encore brièvement que le thème du jardin est omniprésent dans l'art pictural des enluminures persanes ${ }^{11}$ chez Gunayd (XIVeme s.), Behzād (XVème s.), Mirak (XVème s.) et tant d'autres, pour ne mentionner que les plus anciens. Un rapprochement est également à faire entre le jardin peint par les artistes persans et les descriptions dans Les Mille et Une Nuits. ${ }^{12}$.

\section{Les vocables coraniques}

Le vocable de loin le plus fréquent dans le Coran pour désigner le jardin est ganna, employé 66 fois au singulier, 69 fois au pluriel (gannăt) et 7 fois au duel (ğannatān [3] ou gannatayn [4]). La quasi totalite de ces attestations renvoie au jardin du paradis ${ }^{13}$. C'est en premier lieu le jardin où séjourna $A_{d a m^{14}}$. Les musulmans irréprochables en seront propriétaires (ashăb). L'expression așäb al-

géographe arabe Yāqūt (1179-1229) évoquent encore très nettement la réalité du jardin sassanide, appelé firdaws en persan. N'oublions pas que la tradition zoroastrienne (Zoroastre: 660 av. J.C.) promet, dans l'Avesta, que les élus se retrouveront dans un jardin-paradis, plein de bonnes odeurs et d'une lumière éternelle. Ce paradis est entouré d'une enceinte qui en persan s'appelle: pairi-daeza! Le jardin-paradis sassanide modèle est un plan rectangulaire divisé en quatre parties ( $(a h a r-b a g h$ ) par deux axes (allées principales) qui se croisent. Dans les traditions les plus anciennes de l'Asie, le monde est divisé en quatre zones, grâce (souvent) à quatre fleuves. Au centre se trouve une montagne, comme dans les jardins sassanides le palais ou le pavillon du roi...

${ }^{11}$ Dès l'époque timouride (XIVème $s$.) on voit apparaître dans la peinture le thème des bagh que la tradition moghole a si admirablement développé (cf. les jardins de Akbar, Bābur et son petit-fills Akbar...).

12 Nous avons traité ce thème dans une communication faite à Strasbourg en 1994, "Le rôle du portrait et de la représentation dans Les Mille et Une Nuits", lors d'un colloque organisé par les départements des études arabes et iraniennes.

${ }^{13}$ Paradis est une déformation du mot grec paradeisos; mais ce mot vient du vieux persan pairi daeza qui donne également firdaws (que certains font dériver de paradeisos). Le vocable pairi daeza désignait d'immenses parcs où les princes achéménides venaient chasser.

${ }^{14} 2,35 ; 7,19 ; 7,22 ; 7,28 ; 7,44 ; 7,46 ; 7,50 ; 10,26 ; 11,23 ; 11,108 ; 20,121$; 20,117 . 
ğanna revient en effet 9 fois $^{15}$. Il est le jardin de l'immortalité, gannat al-huld $^{16}$, le jardin de délice, gannat al-na ${ }^{\prime} m^{17}$ (au pluriel, l'expression gannat al-na'tm est plus fréquente) ${ }^{18}$, le jardin du refuge, gannat al-ma' $w a^{19}$, le jardin d'Éden, gannat 'adan ${ }^{20}$, le jardin du Paradis, gannat al-firdaws ${ }^{21}$, le jardin sublime gannat 'aliya $a^{22}$ ou encore le jardin, al-gannat, promis à ceux qui font le bien (șălihăt) ${ }^{23}$, ou "les jardins sous lesquels coulent les ruisseaux"24. Dans ce/ces jardins se trouvent des sources, 'uyün ${ }^{25}$, des salles (guraf $)^{26}$, des demeures agréables (masakin țayyiba) ${ }^{27}$. La représentation coranique précise que ce jardin paradisiaque a la grandeur du ciel et de la terre réunis $^{28}$ et se trouve près du jujubier de la Limite (sidrat almuntaha $)^{29}$. L'intérieur est bien ombrage ${ }^{30}$ et parcouru de ruisseaux

152,$82 ; 7,42 ; 25,24 ; 36,55 ; 46,14 ; 46,16 ; 59,20 ; 68,17 ; 78,17$.

${ }^{16}$ Une seule attestation: 25,15 .

1726,$85 ; 56,89 ; 70,38$.

${ }^{18} 5,65 ; 9,21 ; 10,9 ; 31,8 ; 22,56 ; 37,43 ; 52,17 ; 56,12 ; 68,34$.

${ }^{19}$ Deux attestations au singulier 53,$15 ; 79,41$ et une au pluriel: ğannăt al-ma 'wa: 32,19 .

${ }^{20}$ Dans cette expression, le vocable est au pluriel: 9,$72 ; 13,23 ; 16,31 ; 18,31$; 19,$61 ; 20,76 ; 35,33 ; 38,50 ; 40,8 ; 61,12 ; 98,8$.

21 Une seule attestation 18,107 .

2269,$22 ; 79,22 ; 88,10$.

${ }^{23} 2,266 ; 2,214 ; 3,132 ; 3,142 ; 3,185 ; 4,124 ; 7,43 ; 7,49 ; 9,111 ; 13,35$; 16,$32 ; 17,91 ; 18,39 ; 19,60 ; 19,63 ; 26,90 ; 29,58 ; 36,26 ; 39,73 ; 39,74 ; 40,40$; 41,$30 ; 42,7 ; 43,70: 43,72 ; 47,6 ; 47,15 ; 50,31 ; 57,21 ; 76,12 ; 81,13$.

${ }^{24} \mathrm{Au}$ pluriel seulement: gannăt tă̆rt min tahti-hă al-anhār: 2,25; 3,15; 3,$136 ; 3,195 ; 3,198 ; 4,13 ; 4,57 ; 4,122 ; 5,12 ; 5,85 ; 5,119 ; 9,72 ; 9,89 ; 9,100$; 14,$23 ; 22,14 ; 22,23 ; 25,10 ; 47,12 ; 48,5 ; 57,12 ; 58,22 ; 61,12: 64,9 ; 65,11 ; 66,8$; 85,11 .

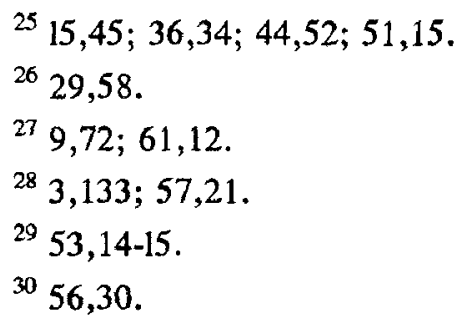


d'eau courante ${ }^{31}$ (má'in gayri äsinin), de lait (laban), au goût non altéré, de vin (hamr) véritable volupté, de miel ('asal) purifié ${ }^{32}$. Ces premières connotations qui pourraient faire penser à une oasis bien arrosée semblent néanmoins sous entendre un ou des palais. D'autres détails du Coran le confirment.

Si ganna l'emporte très largement dans le Coran, d'autres vocables sont à relever comme "le parterre fleuri" (rawda), au singulier ${ }^{33}$ et au pluriel dans l'expression "les parterres fleuris des Jardins" (rawda al-gannât $)^{34}$, la demeure du Salut (där al-saläm) ${ }^{35}$. Or le vocable rawda évoque un jardin à l'intérieur d'un grand palais. Ce paradis possède des portes avec des gardiens ${ }^{36}$. Tout semble donc sous-entendre qu'il y a dans ce paradis des palais merveilleux dans lesquels sont installés les fameuses Houris ${ }^{37}$, mais aussi les élus richement habillés. Ils porteront des bracelets (asãwir) en or $^{38}$, en $\operatorname{argent}^{39}$, en perles ${ }^{40}$. Ils seront revêtus de vêtements en soie, de couleur verte ${ }^{41}$ et seront appuyés sur des coussins verts ${ }^{42}$, des tapis en brocart ${ }^{43}$, des tapis élevés au-dessus du sol ${ }^{44}$. Le Coran mentionne

3156,31 .

${ }^{32} 47,15$. Ce passage fait songer, dans la Bible, à la Terre promise où coule le lait et le miel. Dans la tradition musulmane, quatre fleuves coulent dans le Paradis, chacun étant composé d'eau, de vin, de lait et de miel. Il y a également quatre monts: Uhud. Sinaï, Liban et Hașib.

$3330,15$.

34,22 .

3510,25 . L'idée de paix, salâm, est reprise dans 15,$46 ; 16,32 ; 50,34 ; 56,91$.

3639,73 .

${ }^{37}$ L'expression fi-l-hiyām évoque des pavillons: 55,72 et $37,42-47 ; 56$, 27-37; 88, 9-16.

3822,$23 ; 35,33 ; 18,31$.

3976,21 .

4022,$23 ; 35,33$.

4122,$23 ; 35,33$.

4255,76 .

4355,54 .

4456,34 . 
encore des lits alignés ${ }^{45}$, des lits incrustés d'or et de pierres précieuses $^{46}$, bref, un véritable mobilier de roi. L'idée de palais ou du moins de salle richement meublée est encore évoquée par les séances festives auxquelles auront droit les élus.

En effet, un aspect du paradis qu'il convient maintenant de développer est la boisson ( $(s a r a b$ ), dont les élus jouiront. Cette boisson est un breuvage exquis ${ }^{47}$, pur $(t a h \bar{u} r)^{48}$, limpide $(m a ' \bar{i} n)^{49}$, "volupté pour les buveurs ne contenant pas l'ivresse" 50 , et ne donnant jamais lieu à des disputes ou des bavardages ${ }^{51}$, en totale opposition à la boisson de feu (hamim), que devront boire les réprouvés ${ }^{52}$. Cette boisson exceptionnelle faite à partir d'un mélange de camphre $(k a f i r)^{53}$, ou de gingembre (zanğabrl $)^{54}$, leur est servie par des "éphebes immortels" (wildān) $)^{55}$ ou (gilman) ${ }^{56}$ beaux comme des perles cachees $\left(l u^{\prime} l u^{\prime} \text { maknūn }\right)^{57}$ ou des perles détachées (mantiurr) ${ }^{58}$ qui circulent au milieu d'eux pour leur verser cette boisson dans des vases en argent (äniya min fiḍda $)^{59}$, des aiguières $(a b \overline{a r l q})^{60}$, des cratères

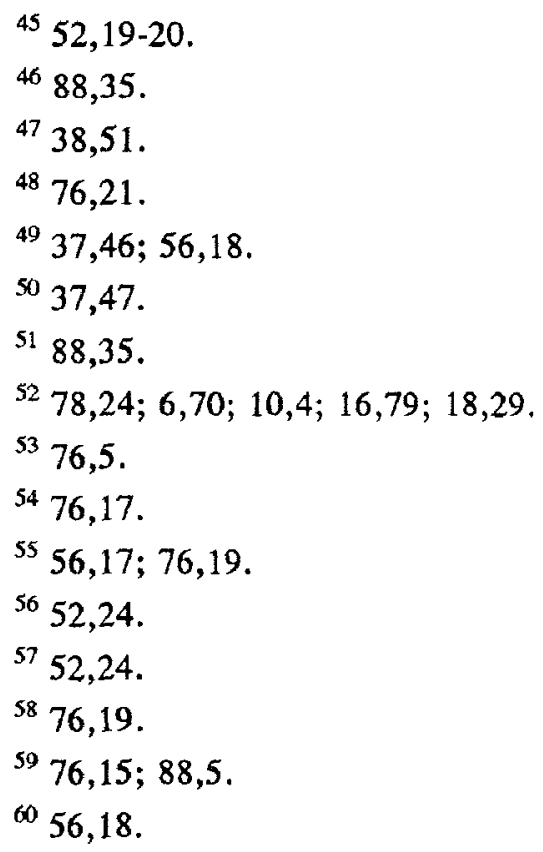


$(q a w a \bar{r} r r)^{61}$, des coupes $(a k w a \bar{b})^{62}$, des verres $\left(k a a^{\prime} s\right)^{63}$. On voit mal les Bédouins accumuler de tels ustensiles dans les oasis.

La plaisir de la boisson est accompagné par le plaisir du manger. Les élus trouveront toutes sortes de fruits en grand nombre ${ }^{64}$, et à portée de main ${ }^{65}$, dans des vergers $\left(h a d a^{\prime} i q\right)^{66}$. Plusieurs arbres sont mentionnés: les palmiers et les grenadiers ${ }^{67}$, les vignes ${ }^{68}$. 11 s pourront également goûter aux viandes fines des oiseaux ${ }^{69}$. Les plats sont en or $^{70}$.

Mais le plaisir le plus subtil reste sans doute la compagnie des fameuses "épouses purifiées" (azwăg mutahhara) ${ }^{71}$, que la tradition a identifiées aux vierges $(a b k a \bar{r})^{72}$, que ni homme ni génie n'a touché ${ }^{73}$. Elles se distinguent par leurs regards modestes, maqsürăat ${ }^{74}$, ou qăsirăt $a l$-tarf ${ }^{75}$. Elles demeurent d'égale jeunesse $(a t r a ̈ b)^{76}$, coquettes $\left({ }^{(u r u b}\right)^{77}$, belles $(h i s a n)^{78}$, comme le rubis et le corail ${ }^{79}$. Elles ont

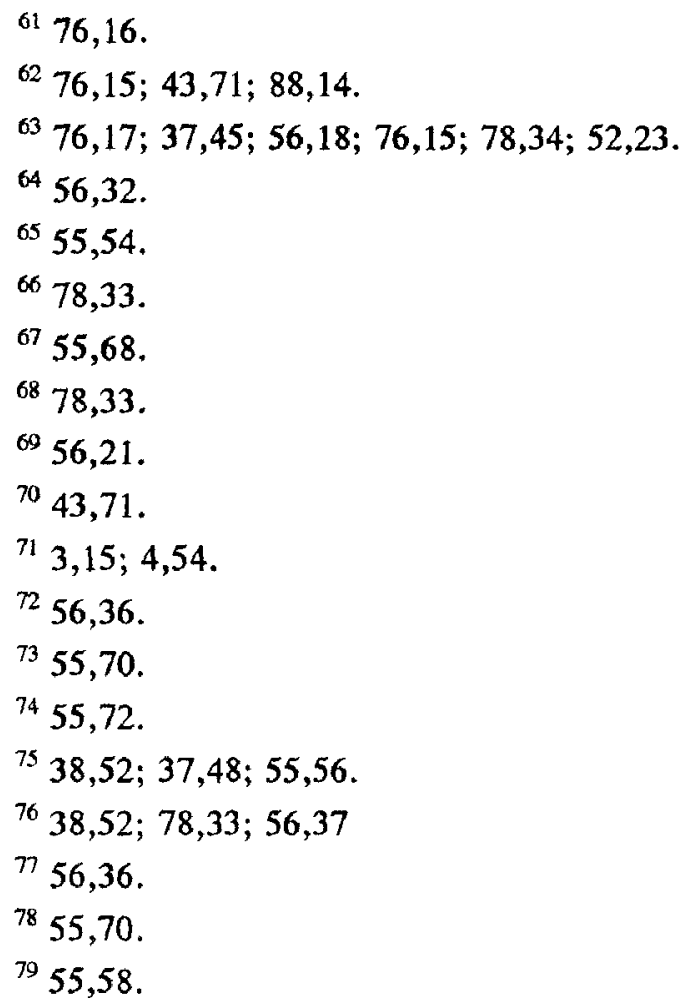


des seins bien formés $\left(k a w a{ }^{\prime} i b\right)^{80}$, des yeux dont le contraste entre le blanc et le noir est très prononcé $(h u ̈ r)^{81}$ (dont on a fait le vocable Houri).

Si nous avons mentionné, dans le détail, une partie de la description paradisiaque, c'est qu'il y a des rapprochement certains à faire avec Les Mille et Une Nuits.

\section{Quelques exemples dans Les Mille et Une Nuits.}

Une remarque cependant s'impose encore: le conteur connaît sans aucun doute les passages du Coran les plus significatifs. Il connaît aussi les immenses palais que les rois et les empereurs se sont fait construire. On peut donc supposer que le conteur intègre à la fois des allusions coraniques et des descriptions faites soit de palais qu'il a vus personnellement soit de reproductions qu'il a pu voir dans la miniature persane. Car, dans l'art pictural persan, figure également le jardin. Dans le Mirăğ Name ou Livre du Voyage du Prophète qui est un manuscrit perse du XVème s. à la Bibliothèque Nationale, on trouve outre la représentation du Grand arbre d'émeraude, aussi le divertissement des Houris au Paradis... dans de splendides jardins. Le conteur est donc capable de faire une savante synthèse entre ces trois principales sources d'inspiration. La description qu'il fait des jardins où évoluent ses héros en sont largement la preuve.

\section{Premier exemple.}

Le premier exemple que nous relevons est la mention du jardin palais dans le conte d'Anis al-Gilis' ${ }^{82}$.

8078,34 .

${ }^{81} 56,22 ; 44,54 ; 52,20 ; 55,72$.

${ }^{82} \mathrm{Cf}$. «L'histoire des deux vizirs et d'Anīs al-Ğilīis» dans Alf layla wa-layla, Dār al-kutub al-'ilmiyya, vol. 1, Beyrouth, pp. 142-163 (s.d.) et aL'histoire de Douce-Amie et d'Ali-Nour» dans Mardrus, Les Mille et Une Nuits, Robert Lafont, vol. 1, 1980, pp. 223-263. 
"Le destin (al-maqadir) les conduisirent [Ali Nūr et son esclave Anīs al-Ǧilīs] dans les jardins (al-basătin $)^{83}$ [de Bagdad]. Ils arrivèrent dans un endroit bien balayé et arrosé. Il y avait de [grands] bancs rectangulaires et des cruches (qawädis) accrochées, remplies d'eau, surmontées d'un dais de roseaux qui se prolongeait tout le long du chemin qui [y amenait]. Au début de ce chemin, [se dressait] la porte d'un jardin, mais elle était fermée... Le jardin s'appelait Jardin de la prédisposition ( $a l-n a z^{\prime} a$ ), dans lequel il y avait un palais (qașr) appelé Palais de la consolation (al-farğa) qui appartenait à Hārūn al-Raš̄ìd... Ce palais avait 80 fenêtres auxquelles étaient accrochées 80 lampes ( $q i n d i l$ ) et à l'intérieur du palais pendait un énorme lustre (sam'adān) en or...

Un jardinier (hüli) gardait ce jardin; c'était un vieillard avancé en âge appelé cheikh Ibrāhīm... Il trouva les deux [jeunes] endormis devant le jardin et couverts avec la même couverture... Il se dit: il n'est pas permis de couper la tête à des jeunes si beaux sans savoir ce qu'il en est... et il les fit entrer dans le jardin.

La porte de ce jardin avait la forme d'un arc (muqantar), couvert d'une vigne aux grappes de differentes couleurs, rouges comme des rubis $(y a \overline{q u} t)$, noire comme de l'ébène (abnūs). Ils entrèrent sous la tonnelle ('ariša) et y trouvèrent des fruits du même genre et d'autres encore. Les oiseaux gazouillaient dans les branches et le rossignol (hazār) chantait; la tourterelle (al-qumri) remplissait de son [roucoulement] l'endroit et le merle sifflait comme [une voix] humaine. Les fruits des arbres avaient muri à point, tous étaient de deux espèces. Ainsi l'abricotier (mišmis) tenait du camphrier ( $k a f u ̈ r i)$ et de l'amandier (lawzi). Il y avait aussi des abricotiers du Khorasan. Les fruits du prunier (barqüq) avaient la couleur des belles (hisān) et les "qaräsi ${ }^{\text {"14 }}$ étonnaient tout le monde par leurs belles couleurs rouges, blanches et vertes. Quant aux fleurs, elles ressemblaient aux perles ( $\left.l u^{\prime} l u^{\prime}\right)$ et au corail (marğăn). Les roses surpassaient en rougeur les joues des belles

${ }^{83}$ Pluriel de bustān qui n'est pas attesté dans le Coran. D'origine persane, ce mot est composé de bū qui signifie: senteur, parfum, odeur, et de la désinence indiquant le lieu: estān. Bustān désigne généralement un jardin potager.

${ }^{84}$ Le vocable al-qaräsiya pourraient être une déformation de karaz: cerise. 
(hisăn). Les violettes étaient pareilles a la flamme du soufre (kibrit) brûlé. La girofle (mantür) et la lavande (huzama) se mêlaient aux anémones. Toutes ces fleurs étaient arrosées par l'eau des nuages. Les corolles des lys [semblaient] sourire et le narcisse regardait la rose avec des yeux noirs et le cédrat (utruß) semblait [porter] des coupes (akwäb) et le citronnier avaient comme des noisettes en or. Toute la terre était tapissée de fleurs diverses. Le printemps arrivait, les ruisseaux murmuraient, les oiseaux gazouillaient et le vent sifflait... Ibrāhīm leur fit servir à manger... et Alī Nür al-Dīn lui dit: je veux du vin (hamr).

Alī Nūr al-Dīn entra dans le cellier (hăsili) et y vit des ustensiles en or (awāni), en argent et en cristal incrustés de toutes espèces de pierreries. Il en choisit ce qu'il voulut et versa le vin dans des carafes (qanänin) et deś pots (bawäti). Sa servante et lui se mirent à boire, émerveillés par la beauté de tout ce qu'ils voyaient...

Ils ne cessèrent de boire, remplis de bonheur, jusqu'à ce que l'effet du vin se fit sentir, que leurs joues se colorèrent, que leurs yeux de gazelles brillèrent et qu'ils dénouèrent leurs cheveux...

Cette longue citation apparaît bien comme un développement des thèmes coraniques. Le vocable coranique ğanna a été remplacé par celui de bustãn qui, lui, n'est pas atteste dans le Coran. En revanche, les fruits [raisin ('inab) 78,33], les oiseaux [tuyūr 56,21], l'eau des ruisseaux [anhar 47,$15 ; 37,46 ; 76,21 \ldots]$, la boisson, les différents contenants du vin $[k a$ 's 56,18 ; akwäb 76,15], faits à partir de l'argent [fidda 76,16; 76,15], le vin lui-même [hamr 47,15], les belles [hisān $55,70 ; 55,76]$, le palais dans son ensemble, le camphrier renvoyant à la boisson faite à partir de camphre [kafür 76,5], les perles [lu'lu' $56,22 ; 52,24 ; 55,22 ; 35,33 ; 76,19]$, le corail [marð̆än 55,22 ]... tous ces vocables appartiennent a la description du paradis céleste. Chez l'auditeur musulman, bercé depuis son plus jeune âge au rythme des versets coraniques, ces mots-clefs ne pouvaient déjà que le renvoyer symboliquement au paradis promis dans l'Au-dela.

\section{Deuxième exemple.}

Les évocations du jardin dans Les Mille et Une Nuits sont très souvent similaires à celle que nous venons de citer. Néanmoins elles ajoutent parfois un élément supplémentaire comme dans le conte d'Alt 
Nūr al-Din et la jeune franque ${ }^{85}$ où le jardin est encore longuement décrit sur trois pages (pp. 461-463).

"Il [Nūr al-Dīn] partit avec eux [ses amis], dans un jardin entouré de hautes et solides murailles. Ce jardin avait une porte voûtée semblable à un iwăn, une porte céleste qui ressemblait à celle du Paradis (al-ğinān) gardée par un portier appelé Riḍwān ${ }^{86}$. Au-dessus de cette porte pendaient des centaines de grappes de raisin de différentes couleurs: rouge comme du corail, noir comme les nez des Soudanais, blancs comme l'oeuf ${ }^{87}$ de pigeon. Dans le jardin se trouvaient des prunes (hawh) et des grenades (rumän), des poires (kummatri), des abricots (barqüq) et des pommes. Toutes ces sortes de fruits avaient des couleurs différentes, ils n'avaient pas leurs pareilles (gayr șinwān $)^{88} \ldots$ Dans ce jardin, il y avait des fruits (fawakih) formidables, des oiseaux de toutes sortes et de toutes les couleurs comme le fahat, le rossignol (bulbul), le courlis (kurwān), la tourterelle (qumari), le pigeon (hamām) [...], des ruisseaux (anhär) d'eau courante (al-mä' al-ğări). Les arbres de ce jardin avaient chacun deux especes de fruits..."

Suit alors quasiment la même énumération de fruits et de fleurs mentionnés plus haut et le conteur de conclure par ces mots:

"Ce jardin n'a pas son semblable, il est comme une partie (qit 'a) du Paradis (ginan)... la langue ne peut pas le décrire à cause des [choses] étonnantes ('ałáa'ib) et étranges (gară'ib) qu'il contient et qui ne se trouvent que dans le Paradis".

Par ce rappel des fruits, le conteur cherche à évoquer une fois de plus les joies de l'Au-dela. L'allusion est même directe dans le vocable ginăn qui, bien qu'absent dans le Coran, renvoie directement au ganna coranique. La porte du jardin en forme d'iwān si elle est traditionnelle dans l'architecture sassanide, iranienne voire des premiers jardins de

${ }^{85}$ «Histoire de Al̄̄ Nūr al-Dīn avec Miryam la ceinturière», dans Där al-kutub, vol. 2, p. 461, et Mardrus: «histoire du jeune Nour avec la franque héroïquen, vol. 2, p. 259.

${ }^{86}$ Dans la tradition arabe (mais non coranique à proprement parler), il est l'ange gardien du Paradis.

${ }^{87}$ Le conteur joue sur les mots avec bayd (oeuf) et abyad (blanc) d'ailleurs forme élalive de bayd.

${ }^{88}$ Dār al-kutub. vol. II, 461. 
Bagdad et Sãmarrā, n'en rappelle pas moins la porte (bāb pl. abwāb) du jardin dans le Coran ${ }^{89}$ que la tradition postérieure a fait garder par des anges ${ }^{\infty}$. La longue énumération des fruits ne fait qu'actualiser l'affirmation coranique "des fruits abondants (fakihatin katiratin)".

Il faut remarquer que le monde arabe aura connaissance des fruits et des fleurs grâce à l'Iran dont la tradition remontre très loin dans le passé, si l'on croit Xénophon décrivant le parc de Cyrus le Jeune (407 av. J.C.) à Sardes. La tradition achéménide sera recueillie par les Sassanides qui la légueront aux Arabes.

\section{Le troisième exemple.}

A travers la beauté des jardins terrestres, les conteurs cherchent donc à évoquer celle du paradis. Le lien entre les deux est explicite dans le conte de lbrähīm et Ğamila ${ }^{91}$. Le héros, le jeune Ibrahīm est introduit dans un jardin de Bașra où vit la fille dont il a vu l'image collée dans un livre.

"Quand Ibrāhīm vit ce jardin il eut l'impression de voir le Paradis [al-ğanna]. Il vit les arbres touffus (multaffa), les palmiers (nahil) géants, les eaux abondantes, les oiseaux qui chantaient différents airs et il s'avança vers la coupole $(q u b b a)$... qui avait quatre portes auxquelles on arrivait après avoir gravi cinq marches. Au milieu se trouvait un bassin (birka) dans lequel on descendait grâce à une échelle en or... et au milieu du bassin il y avait une fontaine (salsabit) en or avec des figurines (suwar) grandes et petites, des bouches desquelles jaillissait l'eau. Quand l'eau sortait de ces figurines, en faisant toutes sortes de bruits, on se serait cru au Paradis (al-ganna)... Le jardinier lui demanda: comment vois-tu mon jardin? Le jeune homme lui répondit:

89 "(49) En vérité, les Pieux auront certes beau lieu de retour: (50) les jardins d'Éden aux portes pour eux toutes ouvertes (51) où, accoudés, ils réclameront des fruits abondants et un [exquis] breuvage, (52) tandis qu'auprès d'eux seront des [vierges] aux regards modestes, d'égale jeunesse. (53) Voilà ce qui vous est promis au Jour du Jugement" Coran. 38,49-53).

${ }^{90}$ Dans le Coran les anges ne gardent pas les portes du paradis. Ils gardent en revanche les "Feuilles vénérées", à savoir l'archétype du Livre: 80,15-16.

${ }^{91}$ Dār al-kutub, vol, 2, p. 613. 
c'est le paradis sur terre (huwa gannat al-duniya).. Le jardinier s'absenta un moment et revint avec un poulet [rôti] et de la nourriture..." (p. 613).

Plus loin dans le conte, lbrāhīm se promène dans ce jardin merveilleux et cueille les fruits qu'il veut manger... jusqu'à ce que le jardinier lui annonce la venue de Ğamila.

"Alors il la vit. Elle portait ses bijoux (hula) et ses robes (hulal), sur sa tête une couronne incrustée de perles (durr) et de pierreries ( gawāhir), à son cou $(\not g a y d)$ un collier de perles $\left(l u^{\prime} l u^{\prime}\right)$, à sa ceinture une topaze (zabarğad) attachée avec des fils de rubis (yãqüt) et de perles $\left(l u^{\prime} l u^{\prime}\right) . .$. Et Ibrāhīm se dit en lui-même: il ne fait point de doute, les portes du ciel se sont ouvertes..." (p. 614).

Dans cet exemple, le vocable coranique al-ganna est employé pas moins de trois fois et la sourate 76 est directement mentionné à travers le nom de la source céleste: salsabil $(76,18)$. L'expression "portes du ciel" est également coranique ${ }^{92}$. La description de Gamila évoque la richesse et la beauté des vêtements des élus comme nous l'avons signalé plus haut. Et Ibrähīm se comporte dans ce jardin comme un élu à qui est donné le droit de goûter aux fruits qu'il veut.

\section{Le quatrième exemple.}

Dans le conte de Qamar al-Zamān et Budūr ${ }^{93}$, le héros Qamar al-Zamän, après qu'un oiseau eut dérobé la bague de sa fiancée Budür, aboutit dans une ville de Mages qu'il traverse et se trouve au milieu des jardins, en bordure de la ville.

"... et il arriva dans les jardins de la ville, continua au milieu des arbres, et atteignit un jardin. Il s'arrêta à la porte, aussitôt en sortit le jardinier qui lui souhaita la bienvenue (rahaba bi-hi) en disant: louange

${ }^{92}$ Fa-fatahna abwäb al-sama': Puis Nous avons ouvert les portes du ciel à une eau torrentielle... $(54,11)$ évoque le déluge dont fut témoin Noé. Le jour du Jugement "le ciel sera ouvert et sera portes [béantes]" $(78,18)$. L'expression revient une nouvelle fois dans un contexte différent du déluge, à savoir le Jugement.

93 «L'histoire du roi Qamar al-Zamānn el de son père Šahramān»: Dār al-kutub, vol. 1, pp. 398-445; Mardrus, vol. 1, pp. 547-605. 
à Dieu qui t'a fait parvenir jusqu'ici sain et sauf (sãliman) [sans être inquiété] par les habitants de cette ville. Entre vite dans ce jardin avant que quelqu'un ne t'aperçoit et Qamar al-Zamān entra...".

La suite du conte nous apprend que le jeune prince se charge d'arroser les arbres, de couper les herbes... jusqu'au jour où il découvre dans le sous-sol un trésor immense rempli d'or et de pierreries. Ce qui retient notre attention dans ce jardin, ce n'est pas cette richesse dont il hérite, ce n'est pas la bague de Budūr qu'il retrouve dans le ventre d'un oiseau... c'est la fonction même du jardin. Qamar al-Zamān est arrivé dans une ville hostile, habitée par des Mages, et le jardinier est le seul musulman de la ville. Le jardin devient alors le refuge naturel de Qamar al-Zamān. Or le paradis du Coran n'est-il pas le refuge (al-ma'wa 32,19; 53,15; 79,41) ou encore la maison du salut (där al-saläm 10,25) où les élus sont accueillis avec la salutation du salam $(7,46 ; 14,23 ; 15,46 ; 16,32 ; 40,34 ; 19,62 ; 25,75$; $56,26)$. Le Refuge, l'espoir suprême de tout croyant et de celui qui écoute les contes pour déjà se l'imaginer sur terre.

Ces quelques exemples suffisent donc à montrer à quel point Les Mille et Une Nuits ne font donc qu'expliciter, en quelque sorte, l'évocation coranique. Mais celle-ci est d'autant plus réelle et concrète que le conteur pouvait aussi faire référence à des réalisations architecturales non seulement fictives mais bien réelles. Les capitales du monde arabe donnaient largement des exemples probants et le conteur n'avait aucun mal à insérer dans son histoire la description d'un palais bien connu par ses auditeurs. II est difficile pour un lecteur d'aujourd'hui de saisir ces sous-entendus auxquels le conteur recourait. Il avait en effet deux grands types de jardins à sa disposition: le jardin extérieur à la ville et celui qui se trouve dans le palais. $\mathrm{Si}$ nous revenons à l'exemple donné dans le conte de Qamar, on constate que le jardin semble bien se trouver en bordure de la ville des Mages, suffisamment en retrait pour qu'il soit séparé de la ville. Les jardins sassanides se trouvaient en effet hors des villes et les bâtisseurs abbassides ne manqueront pas de reproduire ces jardins en les encadrant de divers éléments architecturaux. Les agdals marocains en sont la parfaite imitation. Parfois ils étaient ornés de kiosques et toujours parcourus de canaux. Les Mille et Une Nuits en rendent largement compte. Le deuxième type de jardin est le riyăd ou le jardin intérieur, 
élément constitutif du palais ou des riches demeures. C'est encore de l'ancienne Perse que vient la creation du riyad dont on trouve egalement des traces dans Les Mille et Une Nuits. Mais cette extension de notre propos nous éloignerait des jardins que nous avons évoqués et que le conteur ne se presse jamais de quitter.

${ }^{94}$ Et c'est en Andalousie que l'on peut voir d'illustres réalisalions, malgré les siècles qui séparent les premiers des derniers. L'exemple le plus brillant est la cour des Lions de l'Alhambra du nașrīe Muhammad V (1362-1391) et aussi le célèbre Generalife avec ses canaux et ses jets d'eau. Ces jardins andalous serviront de modèles aux princes maghrébins si l'on considère certains palais de Fes et de Marrakech. Notamment le palais Badi' du saadien Aḥmad al-Manṣūr (1578-1603). 